\title{
Elastomeric Structural Attachment Concepts for Aircraft Flap Noise Reduction - Challenges and Approaches to Hyperelastic Structural Modeling and Analysis
}

\author{
Thammaiah Sreekantamurthy ${ }^{1}$ \\ Analytical Mechanics Associates, Hampton, Virginia, 23681 \\ and \\ Travis L. Turner ${ }^{2}$, James B. Moore ${ }^{3}$ and Ji Su ${ }^{4}$ \\ NASA Langley Research Center, Hampton, Virginia, 23681
}

\begin{abstract}
Airframe noise is a significant part of the overall noise of transport aircraft during the approach and landing phases of flight. Airframe noise reduction is currently emphasized under the Environmentally Responsible Aviation (ERA) and Fixed Wing (FW) Project goals of NASA. A promising concept for trailing-edge-flap noise reduction is a flexible structural element or link that connects the side edges of the deployable flap to the adjacent main-wing structure. The proposed solution is distinguished by minimization of the span-wise extent of the structural link, thereby minimizing the aerodynamic load on the link structure at the expense of increased deformation requirement. Development of such a flexible structural link necessitated application of hyperelastic materials, atypical structural configurations and novel interface hardware. The resulting highly-deformable structural concept was termed the FLEXible Side Edge Link (FLEXSEL) concept. Prediction of atypical elastomeric deformation responses from detailed structural analysis was essential for evaluating feasible concepts that met the design constraints. The focus of this paper is to describe the many challenges encountered with hyperelastic finite element modeling and the nonlinear structural analysis of evolving FLEXSEL concepts. Detailed herein is the nonlinear analysis of FLEXSEL concepts that emerged during the project which include solid-section, foamcore, hollow, extended-span and pre-stressed concepts. Coupon-level analysis performed on elastomeric interface joints, which form a part of the FLEXSEL topology development, are also presented.
\end{abstract}

\section{Introduction}

A irframe noise is the component of total aircraft noise that is not associated with propulsion and is generally attributable to unsteady aerodynamics caused by flow over the airframe. Decades of progress in engine noise reduction has rendered airframe noise an equal contributor to overall noise for typical transport aircraft during the approach and landing phases of flight. Future Federal Aviation Administration regulations will mandate additional, aggressive reductions in aircraft noise in the vicinity of airports. Thus, technology for reduction of airframe noise is crucial for meeting those requirements and, consequently, it has recently been emphasized under NASA's Fixed Wing Project (Ref. 1) of the Fundamental Aeronautics Program and the Environmentally Responsible Aviation Project (Ref. 2-5).

Unsteady aerodynamics caused by the deployed, trailing-edge flap is a particularly prominent airframe noise source for many aircraft. Previous experimental and computational aerodynamic and aeroacoustic work has shown that the pressure difference across the flap, responsible for lift, has a strong tendency to equalize at the flap side edges. That pressure-equalization process results in the formation of an intense vortex system, demonstrated in

\footnotetext{
${ }^{1}$ Engineer 6, AMA, c/o NASA Langley Research Center, M.S. 463, 2 N. Dryden Street, Hampton, VA 23681.

${ }^{2}$ Senior Research Engineer, Structural Acoustics Branch, Mail Stop 463, Senior Member, AIAA.

${ }^{3}$ Senior Research Engineer, Structural Mechanics \& Concepts Branch, Mail Stop 190.

${ }^{4}$ Materials Research Engineer, Advanced Materials \& Processing Branch, Mail Stop 226.
} 
Figure 1, that interacts with the flap solid surfaces at the side edges to produce significant noise. Numerous concepts have been studied to weaken this vortex formation at the deployed flap edge or otherwise mitigate this aeroacoustic noise source (Ref. 3-5). Concepts that have been considered for flap noise reduction include flow-resistive treatments, e.g., porous elements and brushes, and flow-redirection treatments, e.g., fence-type structural modifications. A suitably-shaped structural connection (also termed link or element) that bridges the separation between the deployed flap side edge and the adjoining main wing edge, as seen in Figure 2, has been shown to be among the most promising treatments for flap noise reduction. This structural connection weakens the vortex formation, and thus the flap noise, by delaying the pressure equalization to near the flap trailing edge. Such a structural connection is also among the most challenging to implement because of the large shape change and/or deformation that is required to reconfigure the structural connection between the retracted and deployed configurations.

Previous work to produce a structural connection between the flap side edge and the main wing was focused on a very gradual, gracefully-fared structure that subtended a significant span, as shown in Figure 2. In particular, an extensive effort was undertaken by Boeing (Ref. 6) to develop a Continuous Moldline Link (CML), under the Quiet Technology Demonstrator Program, as a flap side edge treatment for noise reduction. In that effort, design

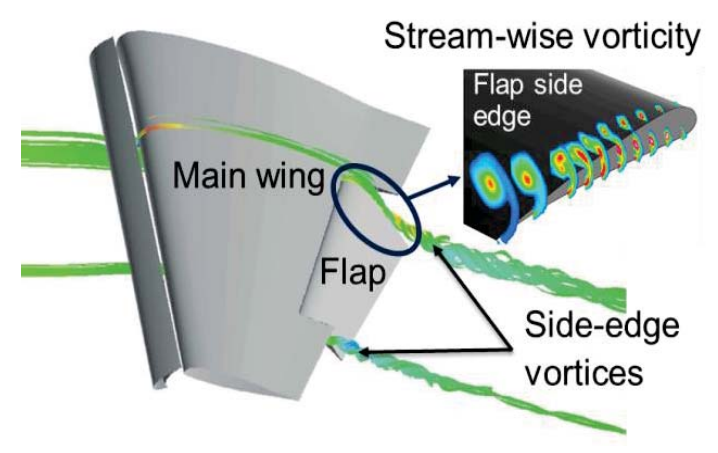

Figure 1. Typical-transport wing with deployed trailing-edge (TE) flap showing side-edge vortices, after Streett et al. (Ref. 3).

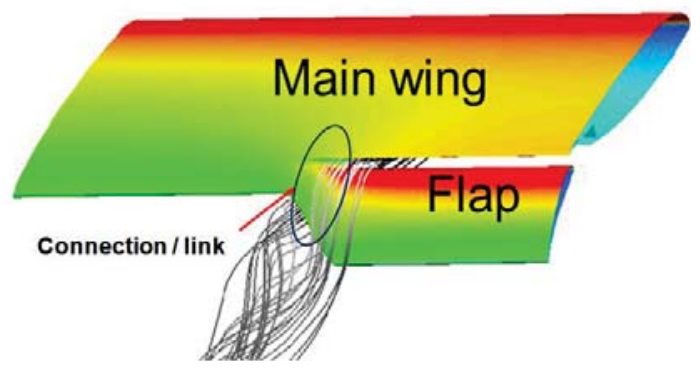

Figure 2. Notional structural connection between the TE flap and main wing subtending significant span, after Streett et al. (Ref. 3). requirements, detailed design, and manufacturing plans were developed for a deforming, flap-side-edge link using the Boeing 777 as the baseline platform. The CML structure, however, subtended a significant span comparable to the flap chord or $\sim 20 \%$ of the flap span (Ref. 6) (Figure 2). A recent Aviation Week article (Ref. 7), notes a longterm and broad collaboration between Boeing and NASA to develop a flexible flap, termed the variable-camber continuous trailing edge flap (VCCTEV), for development of quieter aircraft with efficient high lift in takeoff and landing. More recently, NASA Dryden Research Center, in a joint flight test project with the US Air Force, has undertaken flight tests on a Gulfstream 3 research aircraft for testing an adaptive compliant trailing edge to evaluate shape-changing, bendable composite flaps for simultaneously reducing noise and drag (Ref. 8).

The approach taken in the present work entailed a paradigm shift toward minimizing the span-wise extent of a structural link between the flap side edge and the main wing, e.g., with a span comparable to the flap thickness or $\sim 2 \%$ of the flap span. Minimization of link span has the potential to relieve aerodynamic load and, thereby, reduce the complexity and weight of the structure. Many notional concepts for enabling a short-span link were considered and ranged from purely-mechanized to purely-deforming and combinations of the two. A purely-deforming approach utilizing hyperelastic (elastomeric) materials was selected for detailed study due to its simplicity and potential for reliability, fail-safety and low weight. The structural concept was termed the FLEXible Side Edge Link (FLEXSEL) due to its purely-deforming nature. The FLEXSEL concept is shown applied to a representative single-aisle, transport-class aircraft in the deployed configuration in Figure 3, where FLEXSEL assemblies connect the flap side edge to the main wing at both the inboard and outboard flap ends. It is apparent that the elastomeric link must sustain very large deformation in order to achieve the deployed shape and return to an aerodynamically-clean, cruise configuration. 
The work presented here was part of a broader project that sought to 1) demonstrate the aerodynamic and aeroacoustic performance of static (non-deforming) FLEXSEL assemblies as part of a semi-span, scaled model in the NASA LaRC 14x22 Wind Tunnel and to 2) study the feasibility of designing and fabricating a deformable FLEXSEL structure suitable for experimental flight testing. Substantial development effort resulted in a successful static, wind-tunnel test that accomplished the first objective. That prior work formed the foundation upon which progress toward the second objective was initiated, so essential aspects of the wind-tunnel-model development work will be presented in this document. Achievement of the deformable-sructure for the second objective has been very challenging because of material and geometric nonlinearities, very large deformation and atypical material and structural configurations. The goals of this paper are to describe the challenges encountered in the development of a deformable elastomeric structural concept, present the approaches developed to overcome those challenges, and present the progress made toward eventual achievement of the second objective. Several variants of the FLEXSEL concept evolved during the course of this work. The motivation for each variant will be presented, followed by

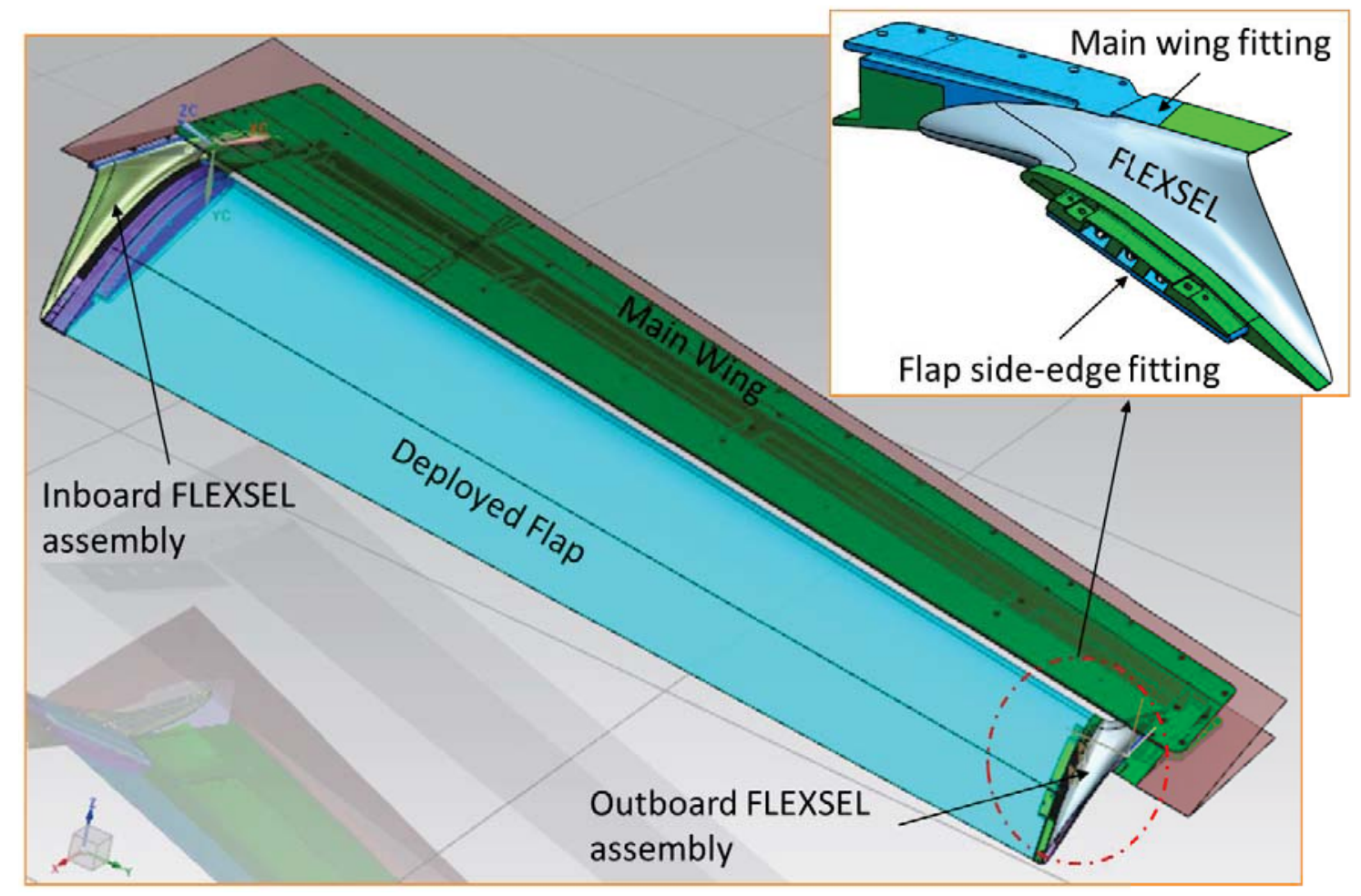

Figure 3. Deployed flap of representative, transport-class aircraft with inset showing FLEXSEL assembly integrated into airframe through transitional fittings at the flap side edge and main wing.

discussion of key analyses and results. Special analysis cases performed on subcomponents essential to development of the concepts will also be shown.

\section{FLEXSEL Elastomeric Structure}

A small, single-aisle, transport-class aircraft was selected for development of the FLEXSEL concept because of the opportunity for testing of a sub-scale, static model in the NASA LaRC 14x22 wind tunnel for aerodynamic and aeroacoustic performance. The general requirements for the structural link included maintaining a clean cruise configuration with minimal drag addition, achieving a high-lift configuration that significantly reduces flap-sideedge noise with either a benefit to the aerodynamics or negligible aerodynamic loss $\left(C_{L}\right.$ vs. $\alpha$ and $\left.C_{L \max }\right)$ and 
maintaining an aerodynamic shape under load in all configurations. These requirements were accommodated by matching the baseline FLEXSEL cross-section to that of the adjoining flap side edge such that the FLEXSEL becomes a prismatic extension of the flap to the adjacent main-wing edge in the retracted/cruise configuration, as shown in Figure 4. Other requirements specific to the chosen airframe included a max weight addition of $100 \mathrm{lbm}$ per wing and a maximum force for deformation of the FLEXSEL structures of $300 \mathrm{lbf}$ per wing, both at full scale.

\section{A. Configuration}

Although it is acknowledged that the structural configuration and airframe integration approach will likely differ at full scale, the existing CAD models and relatively mature design details of the static, wind-tunnel model facilitated parametric study at model scale. Thus, all models and results shown in this study for the deforming FLEXSEL are at the same scale as the static, wind-tunnel model unless indicated otherwise. The baseline FLEXSEL configuration has the aerodynamic shape of the flap section and is stress-free in the retracted configuration, as shown in Figure 5. The dimensions of the baseline FLEXSEL cross section at model scale were $~ 10$ inches in chord, $\sim 1.6$ inches in thickness and 1.5 inches in unsupported span. The unsupported span corresponds to the distance

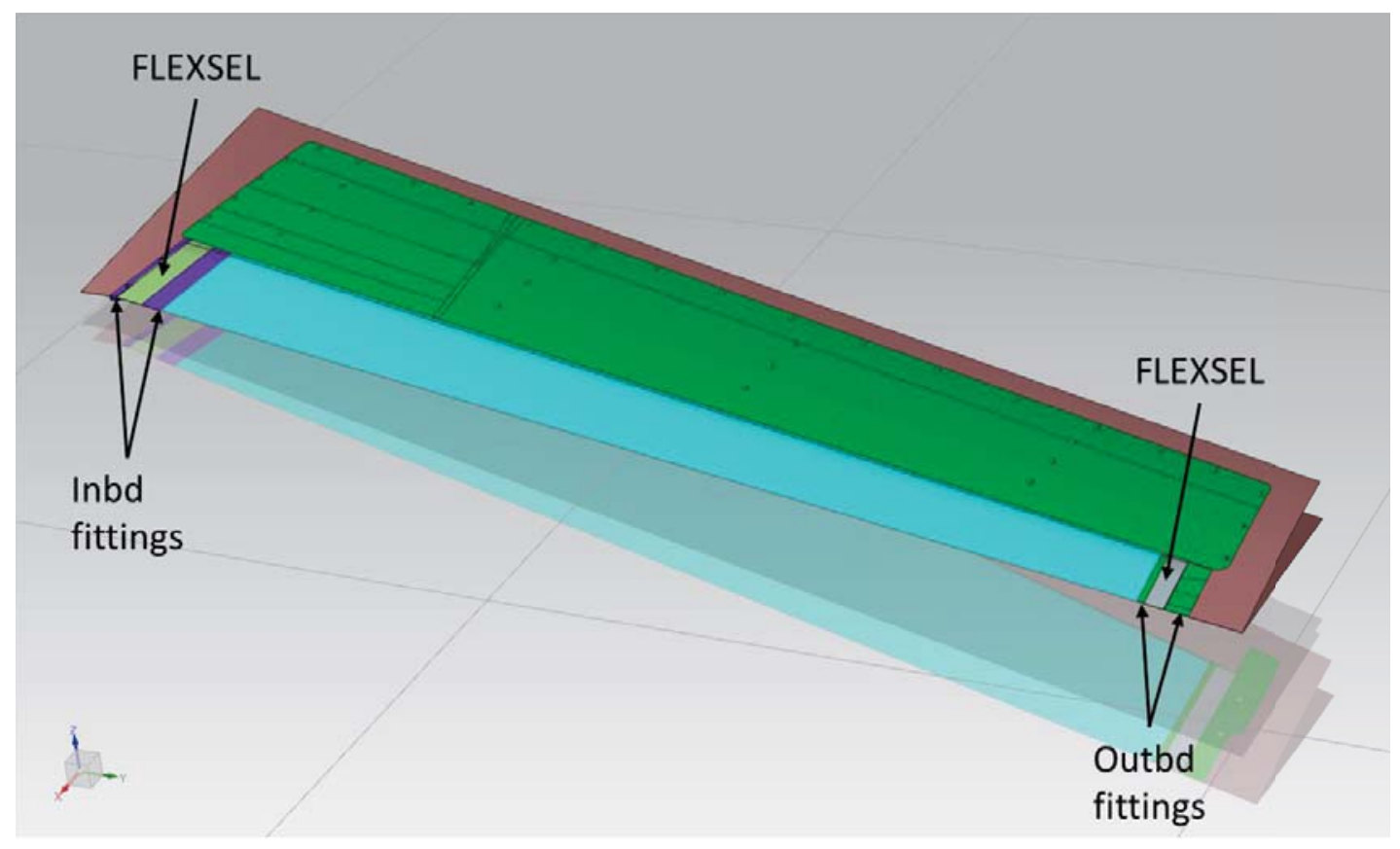

Figure 4. Retracted flap showing baseline FLEXSEL assemblies as prismatic extensions of the flap section to the adjoining main-wing edges.

between the inner edges of the flap and main wing elastomeric attachments.

The FLEXSEL elastomeric structure must deform as the flap is deployed to achieve the deployed configuration depicted in Figure 6. There is a great stiffness and deformation discontinuity where the FLEXSEL elastomeric structure meets the conventional airframe structure. Many options were considered to join the two structures and bridge this discontinuity. All concepts involved integral elastomeric attachments encased within fittings. The selected joining concept leveraged the incompressibility of the elastomer to strengthen the joint and the resulting FLEXSEL assembly is depicted in Figure 6. As load tends to liberate the elastomer from the fitting, the embedded conical inserts push the elastomeric material away circumferentially. The incompressible, volume-conserving nature of the elastomer generates compressive stress between the inserts and fitting walls, where mating tongue-and-groove features also work to retain the elastomer. More details of these joining concepts are described in subsequent sections. 
The flap deploys on the flap track when it is commanded to take flap angles ranging from 0 degrees (flap 0) through the maximum flap angle of 39 degrees (flap 39), as indicated in Figure 7. The FLEXSEL structure deforms during flap deployment because it is attached to the flap side edge and main wing at its ends. An idealization of the deformed FLEXEL structure corresponding to the fully-deployed flap position is shown in Figure 6. It was

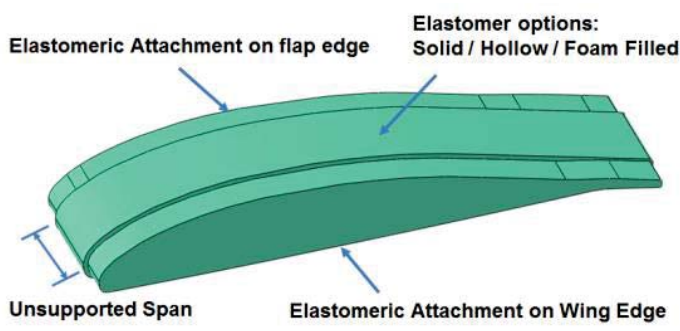

Figure 5. Baseline FLEXSEL without fittings, inserts or retention features.

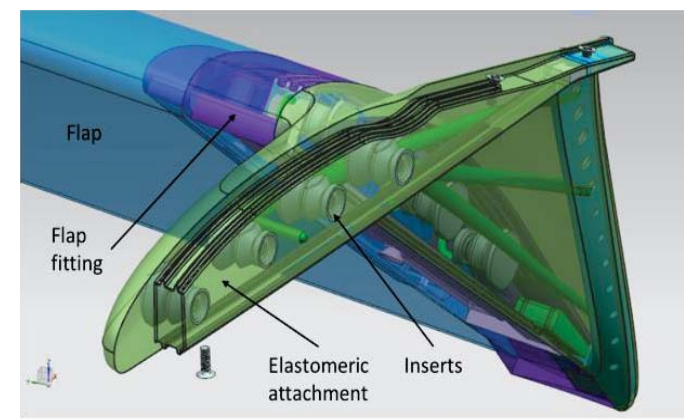

Figure 6. FLEXSEL in deployed configuration with main wing fitting removed showing inserts.

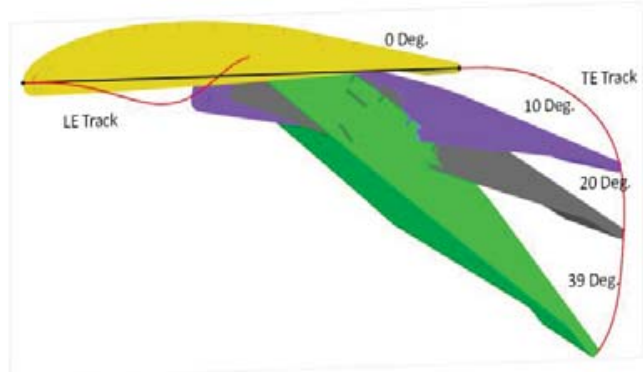

Figure 7. Flap movement for the representative, transport-class aircraft. estimated from the scaled-flap movement that the FLEXSEL structure would have to sustain deformations on the order of $400 \%$ or more in order to achieve the disparate retracted and deployed configurations. This deformation magnitude is challenging even for many elastomeric materials.

Calculations based upon the estimated deformation magnitude and common, elastomer-material response suggested that unreasonable load, on the order of thousands of pounds, would be required to deform a FLEXSEL concept having a solid-elastomeric cross section over the prescribed range of motion. Approaches to reduce this force requirement fall into two categories; those that reduce stiffness and those that reduce deformation. Specific modifications considered in the stiffness-reduction category include selection of soft elastomers, introduction of a hollow cross section and introduction of an elastomeric-foam core. Means to reduce the deformation or strain $(\delta \mathrm{L} / \mathrm{L})$ in the elastomer included increasing the span (L) of the baseline FLEXSEL structure and decreasing the change in dimenion $(\delta \mathrm{L})$ by introducing an intermediate, stress-free shape. The proposed "hollow" and "foam-core" section changes and the "intermediate, stress-free" configuration change for force reduction can be further explained as follows.

A hollow section is achieved by removing the bulk of elastomeric material from the cross section in the unsupported span and leaving only a thin skin. This was identified as a way to greatly reduce the cross-sectional area and, thus, the force developed from the stress on that area. It was anticipated that the hollow FLEXSEL concept could exhibit undesirable deformation, such as wrinkling, unless otherwise constrained. It was hypothesized that an elastomeric-foam material could be substituted in the core to help control such wrinkling and maintain the aerodynamic shape. The foam material could also reduce the force required for deformation and the weight of the structure. The FLEXSEL structure at model scale would then consist mainly of elastomeric foam with a thin, homogeneouselastomeric skin in the unsupported span and solid, homogeneous elastomer in the wing and flap fittings. Other details are shown in the span-thickness section of the retracted FLEXSEL in Figure 8. The hollow FLEXSEL concept is the limiting case of the foam-core approach as the foam density approached zero. 
Recall that the baseline FLEXSEL configuration is stress free in the fully-retracted configuration (flap 0), as shown in Figure 5, and experiences maximum deformation and stress at full deployment of the flap (flap 39, see Figure 6). The force required to achieve the fully-deployed configuration can be further reduced by defining the stress-free shape of the FLEXSEL at an intermediate flap setting between the extreme flap positions of flap 0 and flap 39. This was termed the intermediate, stress-free shape modification to the FLEXSEL concept. The trade-off is that force and deformation are also required in moving the flap from the intermediate position to flap 0 .

\section{B. Material Considerations}

Although a vast body of work exists in development of elastomeric materials and application of them in a wide variety of areas, the availability of suitable elastomeric materials for the FLEXSEL application becomes limited when the specific requirements are taken into consideration such as strength, elastic modulus, large elongation at break, and rubbery plateau over a broad temperature range of $50^{\circ} \mathrm{C}$ to $65^{\circ} \mathrm{C}$. Although soft in constitutive response, the material must also exhibit sufficient toughness and deformation relaxation characteristics suitable for the flap movement rate. Secondary requirements include processing properties such as low viscosity, long pot life (time for mixture to solidify) and good adhesion/cohesion. Operational properties, such as chemical (fuel, oil, deicer, etc.) and environmental (UV, water, etc.) are also germane to the application.

A survey of commercially-available materials was conducted. Material classes that exhibit properties relevant to the FLEXSEL application include silicones, fluorosilicones, polyurethanes and natural or vulcanized rubber. Although silicone and fluorosilicone material classes are likely to offer the best candidates for the application, a polyurethane material (Eager Plastics EP1150) and vulcanized rubber were the focus in this study. EP1150 was selected because of the availability of tensile-test data and because it was felt that the constitutive response represented the stiff end of the relevant material range. Although stressstrain data from a variety of test conditions is required for calibration of the hyperelastic material models in all stress states, only uniaxial test data was available for the EP1150 material (Ref. 9). Vulcanized rubber was selected because of comprehensive material property data available in the literature (Ref. 10-11) and because it was felt that the constitutive response was at the soft end of the relevant material range. Representative tensile-test data from these two materials is shown in Figure 9. It can be seen that the vulcanized rubber material exhibits a much softer and nonlinear constitutive response relative to the EP1150 material.

Elastomeric foams were of interest in this study to potentially serve as a core material for reduction of straininduced loads and for reduction of specific weight. Another

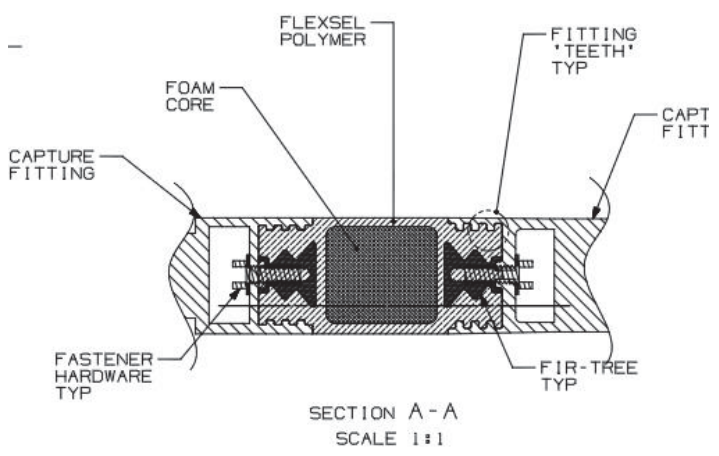

Figure 8. Span-thickness section of foam-core FLEXSEL concept in the retracted configuration.

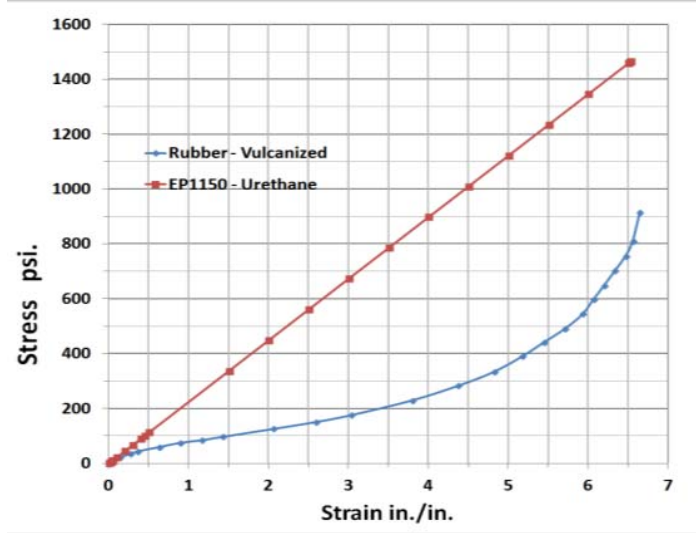

Figure 9. Uniaxial stress-strain response of EP1150 and vulcanized rubber materials.

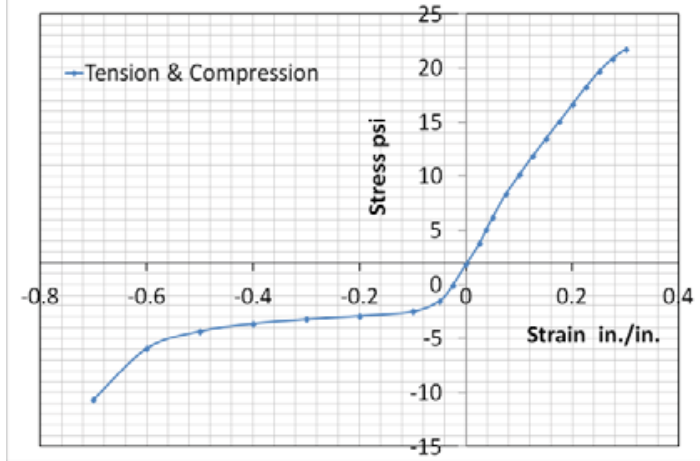

Figure 10. Uniaxial stress-strain data for EF4005 foam material in tension and compression (Ref. 12). 


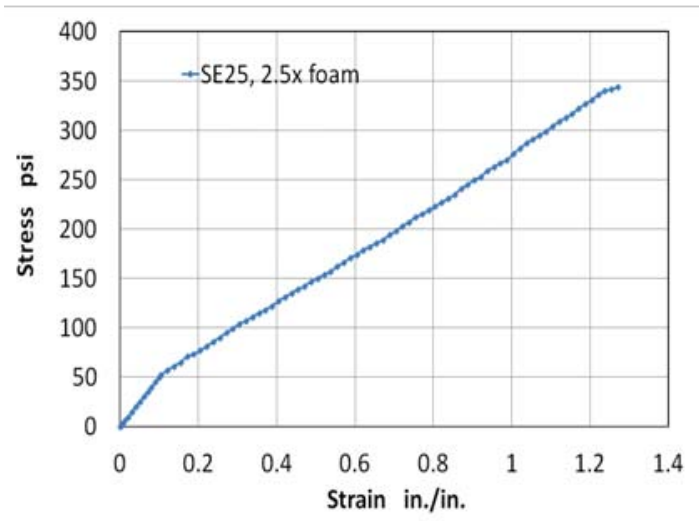

Figure 11. Uniaxial stress-strain data for SE25 foam material in tension. goal of the foam core approach was to help retain the airfoil cross-sectional shape and reduce wrinkling during deformation of the FLEXSEL structure having a thin, homogeneous elastomeric skin. Several elastomeric-foam materials were identified as possible candidates. Sample constitutive responses from two elastomeric-foam candidates are shown in Figure 10 and Figure 11. Mechanical properties of foam EF4005 (Figure 10) were gathered from the material manufacturer's technical data sheet (Ref. 13). It can be seen that the constitutive response of EF4005 in tension is different from that in compression. Furthermore, the response is very soft over a large portion of the compressive strain range. This trend is attributable to the foam permitting large volumetric deformation (cell collapse), which is undesirable for the goal of shape retention. Additionally, only a small amount of tensile strain

can be sustained without failure. This is also undesirable because the foam core is anticipated to experience large tensile strain in the application. Uniaxial tensile properties of flexible foam SE25 (Figure 11) were obtained from inhouse laboratory tests. Although the SE25 material permitted larger tensile deformation than the EF4005, the allowable strain range still did not meet the requirement and the tensile constitutive response of the SE25 was stiff relative to the homogeneous elastomers under consideration (Figure 9). This latter factor suggested that the SE25 elastomeric-foam materials did not offer the intended compliance advantage for deformation-induced force reduction in the application. A few other commercially-available, elastomeric-foam materials may have more desirable properties, but their basic constitutive responses have to be tested and explored further for this application. This was left for future work.

\section{Detailed Modeling Considerations and Approach}

Detailed analysis of the FLEXSEL structure was initiated with the following two prioritized goals: 1) perform a parametric study of the FLEXSEL concept to determine if the strain-induced load requirement could be achieved and 2) determine if the airframe integration approach, involving elastomeric attachments, fittings and inserts (adopted from the static, wind-tunnel model, see Figure 6) was adequate for a deforming model or make necessary modifications to the static-model design. The nature of the modeling fidelity and approach for achievement of these

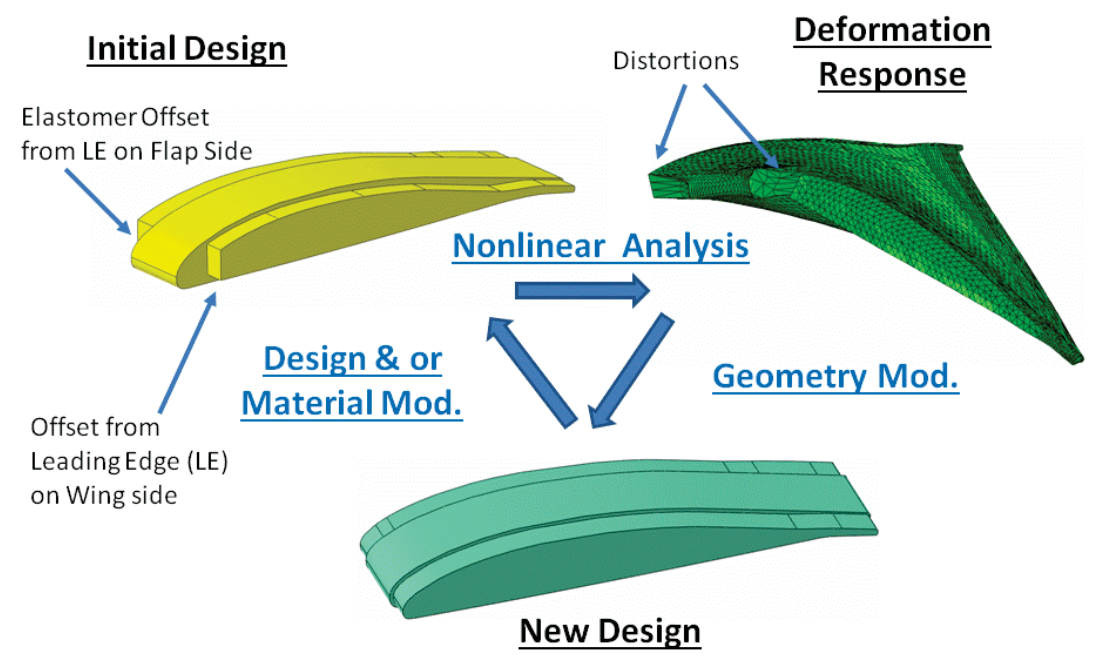

Figure 12. Schematic of an example design cycle encountered in analysis of the FLEXSEL concept.

two goals was necessarily different, as will be explained below.

The FLEXSEL elastomeric structure is asymmetrical in both geometry and loading, which makes simple hand calculations of large deformation responses unsuitable for initial design estimates to satisfy the design constraints. 
As a result, detailed finite element structural analysis was necessary to estimate the very large deformation responses encountered. SIMULIA Abaqus FEA was used in the simulations presented in this study. For example, the initial design for the elastomeric attachment called for truncation near the leading edge to facilitate fabrication of fittings. However, simulation of this configuration under load revealed undesirable distortion of the FLEXSEL at the leading edge, as shown in Figure 12. The response estimates become more involved when material nonlinearity and combination of materials are present. This makes the structural analysis and the design of the FLEXSEL elastomeric

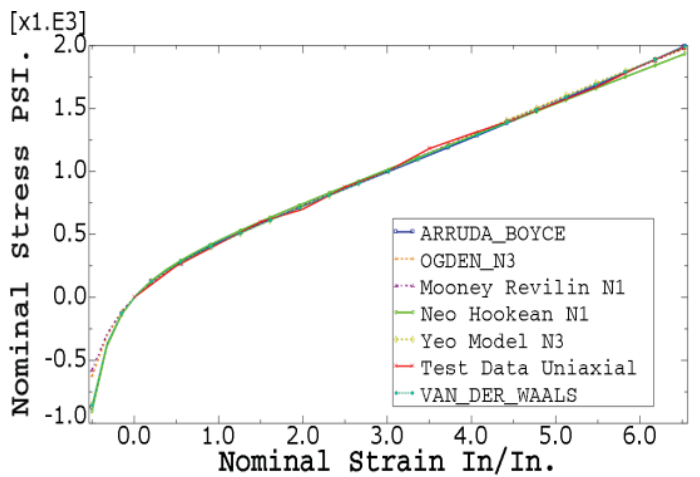

Figure 13. Elastomeric material model comparison with uniaxial test data for EP1150.

structure a highly-iterative process.

One of the challenges in performing analysis of FLEXSEL concepts using FEA tools was the lack of literature relevant to structures exhibiting deformations of the order considered here. Analysis was further complicated by geometric, material, adhesion and contact nonlinearities. Analysis often required models of complicated joints with intricate details that required large mesh sizes. The analysis of FLEXSEL elastomeric models with nonlinear and asymmetrical features often resulted in ill-conditioned numerical computations leading to divergence. A buildingblock approach was used because of these complicating factors. Prismatic blocks in tension, compression, or combined shear and tension loading were analyzed first, followed by analysis of a single elastomer-retention insert joint. Special analysis techniques were explored including Adaptive Meshing, Arbitrary Langrangian-Eulerian (ALE) techniques and axisymmetric elements in an effort to reduce computational complexities. As a result of the above observations, models of varying fidelity for the whole assembly and parts thereof were developed for the FLEXSEL concept to assess the requirement for detailed design, e.g, sections D and E below.

\section{A. Hyperelastic and Hyperfoam Material Modeling}

The deformation response of elastomers is highly nonlinear and cannot be defined by Young's modulus. Further, it is known that the ratio of bulk modulus to shear modulus for elastomers is in the order of $10^{3}$. Thus, the response of an elastomer in shear is primarily determined by shear modulus. Also, the elastomer material is nearly incompressible, but it can undergo large shear when the material is not in a confined volume. It was important to consider these material behaviors when modeling elastomers and interpreting results.

Conventional approaches to modeling the constitutive properties of elastomeric materials include idealized mathematical models and empirically derived equations. Several strain-energy models available in Abaqus were applied to represent a wide variability of stress-strain states of the EP1150 elastomer. In order to select a suitable material model for the EP1150 material, a single-element analysis was conducted to compare the various material models with the test data.

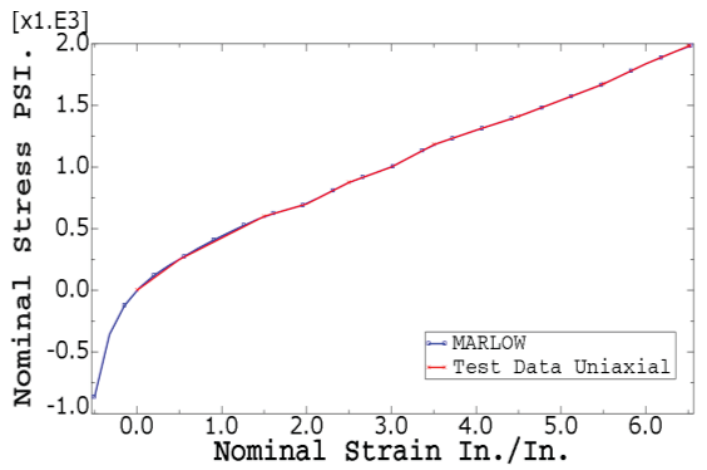

Figure 14. Comparison of Marlow model with uniaxial test data for EP1150
Figure 13 shows a comparison the EP1150 material uniaxial test data with those predicted by the various strainenergy models including Ogden, Arruda_Boyce, and various Polynomials (Ref. 11), all of which captured the test data reasonably well. In addition to these material models, the Marlow model was also evaluated with a single element analysis (Figure 14). The Marlow model assumes that the strain energy potential is independent of the second deviatoric invariant $\mathrm{I}_{2}$. In this model, the deviatoric behavior is defined by specifying uniaxial, biaxial, or planar test data, although only uniaxial test data was available for the EP1150 material in this study. Volumetric behavior was defined by specifying the Poisson's ratio of 0.5, i.e., to model EP1150 as incompressible. The strain energy potential from the Marlow model reproduced the test data,

as expected. The Marlow model was selected for further analysis because of the limited test data. Figure 15 and 


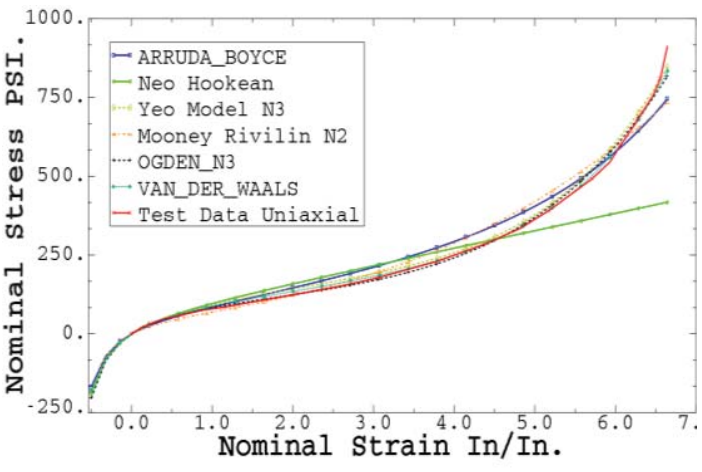

Figure 15. Elastomeric material models of rubber behavior

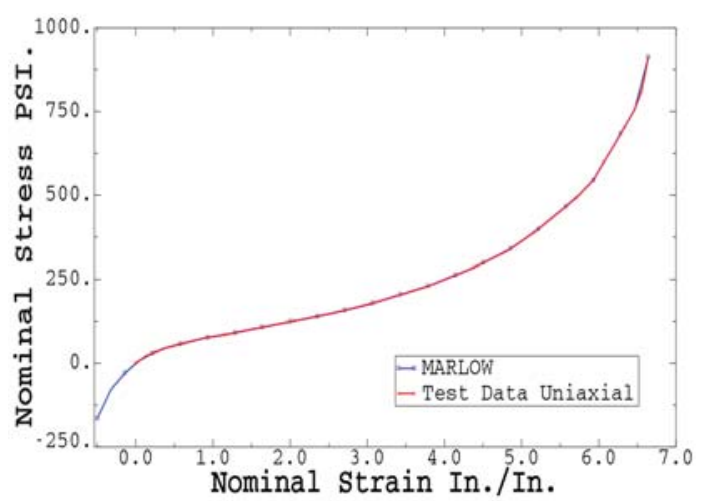

Figure 16. Marlow model of rubber behavior
Figure 16 show the material stress-strain predictions from the Marlow model for the vulcanized rubber material using published uniaxial and biaxial test data. Drucker material stability checks (Ref. 11) were performed on the Marlow model for the vulcanized rubber material case and the model was found to be stable for all load types and strains.

Elastomeric-foam material, in contrast to homogeneous elastomer, can undergo large volumetric deformation. Also, most of the commercially-available flexible foam materials were found to have much less elongation capacity than required in order for those foam materials to be considered for the application. As in homogeneous-elastomer material modeling, an equivalent-strain-energy-based model for estimating the flexible foam material characteristics of Abaqus was used. However, the foam material characteristic parameters required for modeling the strain energy were estimated based on limited uniaxial tensile tests on foam coupons. Also, the foam material characteristics available in references (Ref. 12, 13) were used for preliminary evaluation of the basic responses of the foam core concept. Experimental material data from uniaxial tests of EF-4005 Hyper-foam were interpolated into a second-order strain energy polynomial Figure 10).

\section{B. Contact Surface Modeling}

The FLEXSEL assemblies contained intricate details that created a complex structural joint. Prediction of the joint strength, failure mechanisms and likey need for design iterations required models of sufficient fidelity to capture the relevant behavior. Specifically, load tending to liberate the elastomeric attachment of the FLEXSEL assembly produces deformation around the elastomer-retention inserts

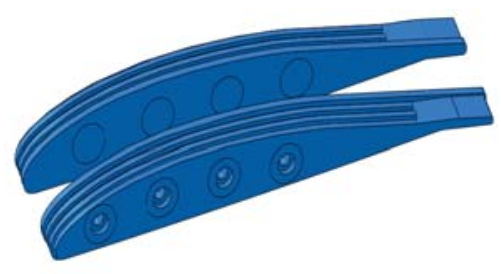

(a)

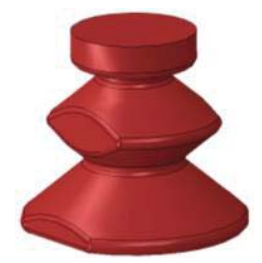

(d)

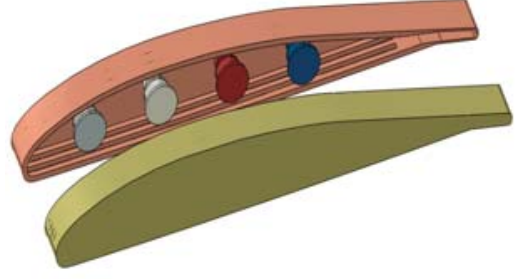

(b)

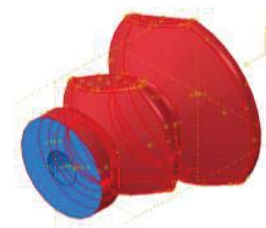

(e)

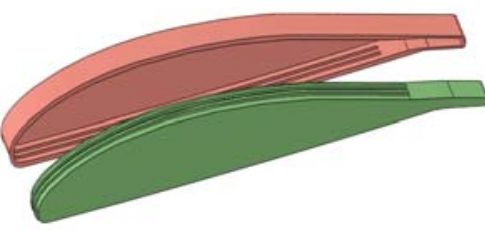

(c)

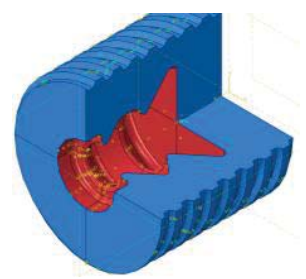

(f)

Figure 17. FLEXSEL elastomeric attachments, fittings and inserts: (a) grooved elastomeric attachments with insert holes, (b) fittings with attached inserts, (c) bonded attachment sleeve, (d) metallic insert, (e) contact surface on insert and (f) contact surface inside elastomer. 
and generates compressive stress within the fitting. That stress works between the inserts and mating tongue and groove features in the fittings and on the elastomeric attachments to provide an interference condition, thereby providing two load paths and greatly increased joint strength.

Figure 17 demonstrates these features of the structural joint. Figure 17c shows the bounding surfaces of the fittings with the tongue and groove features, which was considered as a comparison case. A solid model of a single elastomer-retention insert is shown in Figure 17d. Interaction of a single insert with the surrounding elastomer was isolated in a greatly simplified arrangement represented by the geometry and contact surfaces (red) in Figure 17e and $\mathrm{f}$. Behavior of the elastomer around a single insert and the strength of this simplified joint was studied in this manner. Contact interaction properties assigned for these surfaces were based on Coulomb friction (2-30\%) to model contact forces in the tangential directions and "hard" contact with penalty constraint enforcement was used to model the contact forces in the normal direction.

\section{Flap-Link Mechanism Modeling}

The movement of the flap must be simulated accurately in order to predict the deformation of and the force developed in the FLEXSEL structure throughout the flap deployment. The flap of the representative aircraft was a standard Fowler arrangement consisting of translational and rotational motion. The flap movement was modeled by prescribing the motion of two points; one at the leading edge and one at the trailing edge of the flap, as demonstrated in Figure 18. "Connector" elements were employed to enforce the proper motion and relationship between the two points, as shown in Figure 18 and Figure 19. The manner in which this prescribed motion was imposed on the model varied with analysis intent and the corresponding model fidelity.

Prediction of the load required for deformation of a particular FLEXSEL configuration could be ascertained with adequate accuracy using a simplified model without the wing and flap fittings, i.e., that shown in Figure 5. In this case, the prescribed motion was applied to the elastomeric attachment directly. However, the compliance of the elastomer would not allow for analysis with the aforementioned point-prescribed motion applied to the elastomeric attachment because of excessive local deformation. Instead, it was necessary to prescribe the motion of the section centroid, at the center of the red connector element in Figure 19, and infer the prescribed motion to all nodes on the perimeter of the elastomeric attachment though multi-point

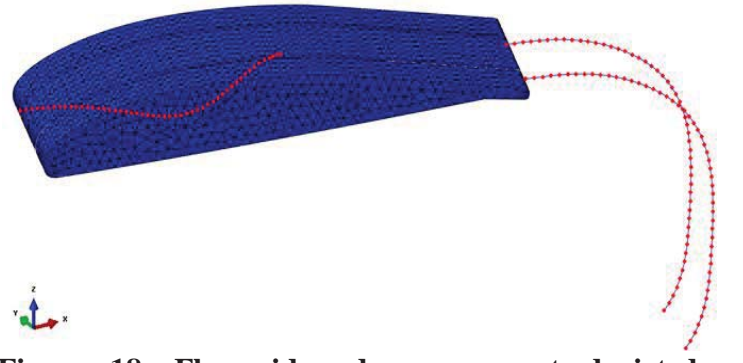

Figure 18. Flap side edge movement depicted on FLEXSEL

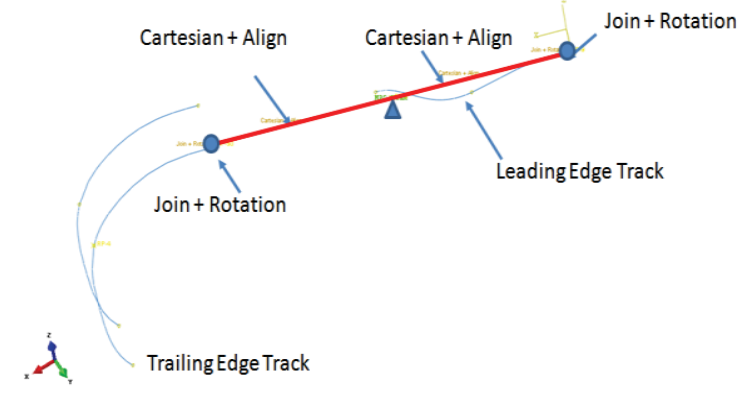

Figure 19. Connector elements enforcing prescribed motion. constraints (MPCs), as indicated in Figure 20. All predictions of the force required for deformation were generated with a model of this type.

Analysis of the deformation within the fittings of the FLEXSEL assembly, i.e., deformation of the elastomeric

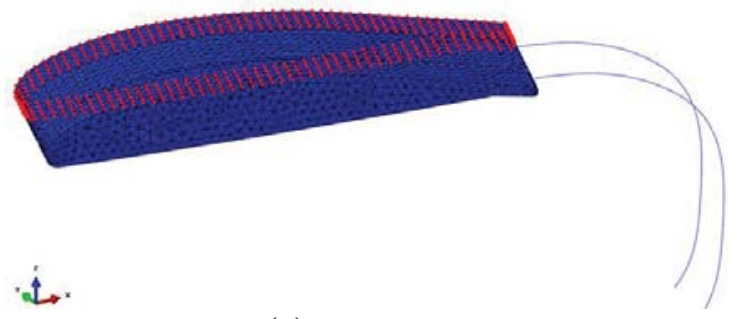

(a)

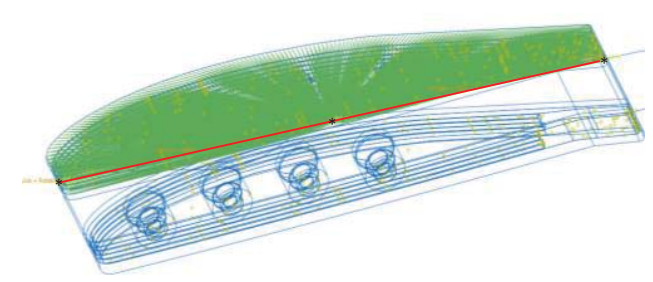

(b)

Figure 20. Elastomeric attachment, connector, and MPC constraint: (a) constrained nodes on the elastomeric attachment perimeter and (b) MPC constraints (radiating green lines) between nodes and centroid of flap chord. 
attachments, and of the strength of the structural joint required significantly greater fidelity than was required for the deformation-induced load analysis. In this case, the FE representation of the fittings and inserts was necessary, along with frictional contact between the elastomer and the retention features (fittings and inserts). The stiffness of the fitting, modeled as a metallic structure, allowed the prescribed flap motion to be applied directly to nodes at the leading and trailing edges of the fitting, respectively. The enforced motion was passed to the elastomeric attachments through contact with the inserts and fitting boundaries. It is noted that a progressive joint failure analysis could be required for detailed analysis of the structural joint consisting of an adhesive bond bridging the material discontinuity until a critical stress defeats the bond, followed by frictional contact transferring the load until the elastomer is failed in cohesion or is liberated from the fitting by excessive deformation (peel mode). These observations are relevant to the discussion in the next two sections.

\section{Elastomeric Joint Analysis}

It was necessary to understand the load-deformation characteristics of a single, elastomer-retention insert in a simplified but representative configuration, i.e., surrounded in elastomer with representative dimensions, etc. before embarking on an investigation of the entire FLEXSEL joint. Figure 21(a), (b) and (c) show a single insert

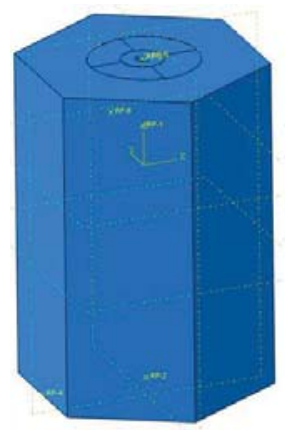

(a)

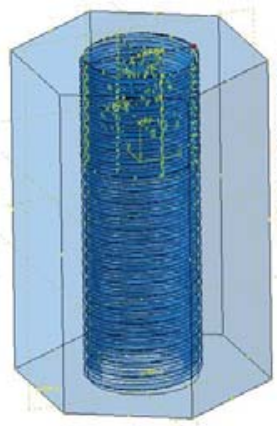

(b)

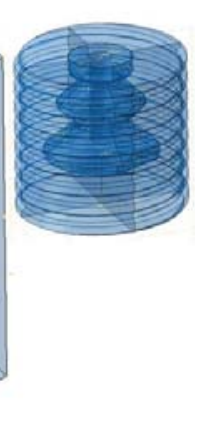

(c)

Figure 21. Simplified elastomeric joint, (a) elastomer and insert housed in a hex-bolt as a support for pull-out test, (b) threads inside hex-bolt as contact surface, (c) insert surrounded by elastomer (molded in hex-bolt).

features (1300 elements of R3D4, R3D3). The large mesh size is due to the fine geometric detail and asymmetry in the insert geometry. Elastomeric joint stresses, strains, nodal reaction forces, and free-body forces were computed in nonlinear analysis cases with contact friction values ranging from $2 \%$ to $30 \%$. For a large but not catastrophic insert movement of 0.23 inches, the elastomeric joint undergoes very large deformations of about $350 \%$ in the joint, as shown in Figure 22. The resultant load versus insert displacement is shown in Figure 22(c) for the 2\% friction case. This plot shows that the maximum load-bearing capacity of the joint is about $80 \mathrm{lbf}$. Increasing the friction to 30\% increased the maximum load capacity to $120 \mathrm{lbf}$. Although large distortions in the FE mesh terminated the simulation at the 0.23-inch displacement, it was felt that the movement of the insert, the deformation in the elastomer and the load generated in the joint was sufficient for the application.

A quarter-symmetry model was also developed to reduce the computational effort and study the element distortion problems. Similar load capacity results were predicted with little improvement in computational surrounded in elastomer and housed in a hex-bolt assembly. Dimensions of the elastomer and thread features in the bolt housing were representative of the application hardware at model scale. The elastomer volume is cylindrical about 1 inch diameter and 1 inch in height. Contact between the elastomer, the hex-bolt and insert was modeled with the same friction formulation mentioned previously. An enforced axial displacement was applied to the insert while the hexbolt was held fixed to study the deformation behavior of the elastomer and to determine the force developed. The FEM of the elastomeric joint included a 3D mesh of the elastomer with linear tetrahedral elements (221885 elements of C3D4H) and a rigid surface mesh hex-bolt (17083 elements of R3D4, R3D3) and insert

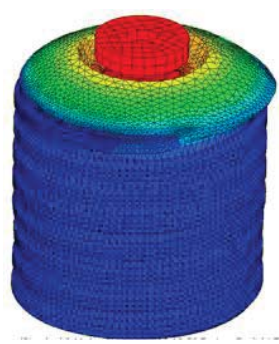

(a)

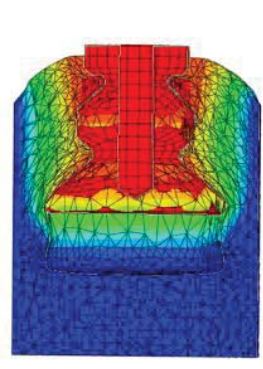

(b)

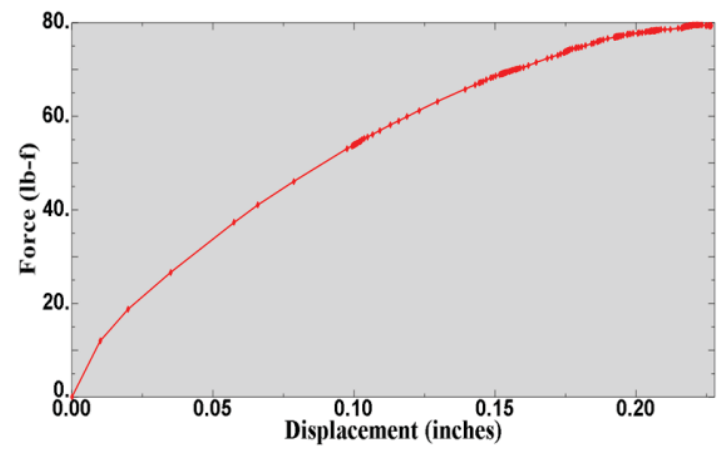

Figure 22. Deformation response of insert in elastomeric joint: (a) elastomer bulging around insert, (b) crosssection of deformed elastomer with embedded insert and (c) reaction force vs. insert displacement. 11

American Institute of Aeronautics and Astronautics 
stability. An axisymmetric model was then developed, which corroborated the load-displacement results and allowed simulation of the insert movement to complete liberation from the joint.

\section{E. Solid-Section FLEXSEL with Detailed Attachment Features}

Analysis of the solid-section FLEXSEL assembly was undertaken, as a full-fidelity model case, to determine the

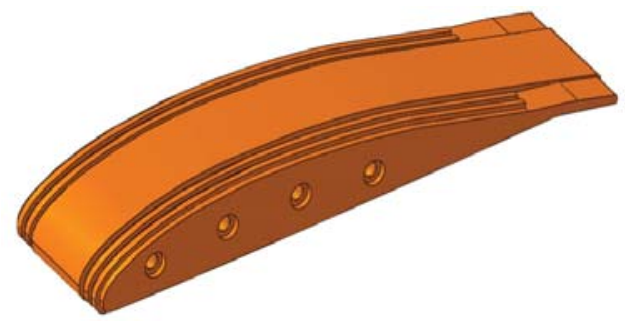

(a)

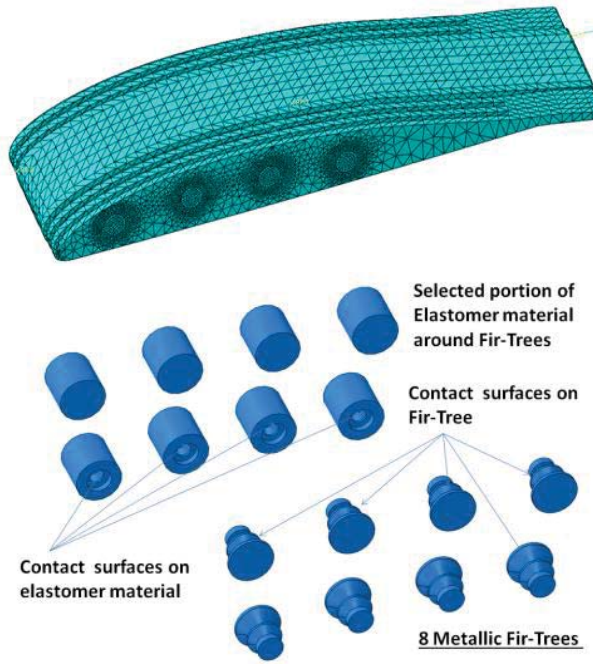
deformation response of the elastomer around the attachment features (fittings and inserts), the effect of that deformation on the shape of the unsupported span and the load-bearing capability of the detailed FLEXSEL joint. Attachment details included tongue and groove features on the elastomeric attachment and the metallic fittings. The elastomeric-retention inserts, embedded in the elastomeric attachments and affixed to the fittings, were also included in the model. A detailed FE model of the solid-section FLEXSEL assembly was developed (Figure 23). The detailed model had 338,422 linear tetrahedral elements. Contact surfaces and interaction definitions were introduced into the model as described previously. Tuning of analysis parameters resulted in a successful analysis through $73 \%$ of the flap motion, as shown in Figure 24(b).

The model was simplified by changing the interaction between the inserts and the surrounding elastomer to "tie constraints" instead of frictional contact. This helped prevent the large distortions

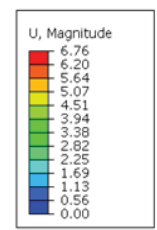

(a)

Figure 23. Solid-section FLEXSEL w/ detailed attachment features: (a) insert holes and tongue-andgroove features on elastomeric attachment, (b) detailed FEM with Fir-Tree inserts and (c) selected portion of elastomer around rows of Fir-Tree inserts.

around the inserts, which allowed for full simulation of the flap movement. The resulting deformations in the elastomeric portion of the FLEXSEL assembly are shown in Figure 24(a). Analysis of the solid-section FLEXSEL consisting of the EP1150 material indicated a maximum free-body force of $\sim 3,500 \mathrm{lbf}$ at full flap deployment. It is clear that $\sim 3,500 \mathrm{lbf}$ is unreasonable for the application, particularly at model scale, and for the joint (elastomeric attachment, inserts, and fittings) design. In fact, the enormous force generated by the solid-section FLEXSEL is the cause for excessive distortion observed around the inserts in Figure 24(b), which was the reason for premature (73\% flap motion) termination of the analysis. Focus was thus turned to a parametric study for a means to reduce the deformationinduced force.
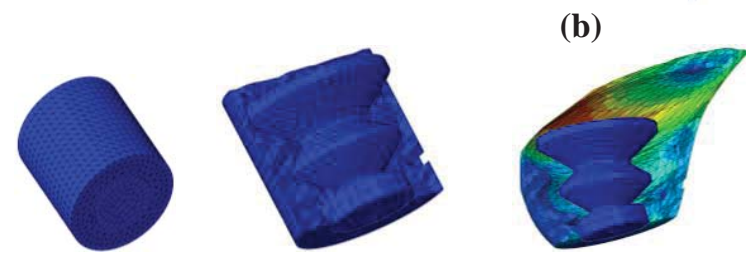

Figure 24. Def. response of solid-section FLEXSEL: (a) insert holes and tongue-and-groove features on elastomeric attachment, (b) selected portion of elastomer surfaces that are either in contact or in separation are shown in undeformed and deformed modes around.

American Institute of Aeronautics and Astronautics 


\section{Parametric Study for Reduction of Required Force for Deformation}

As mentioned at the start of section III, the main goal of this study was to perform a parametric study on known parameters and over allowable ranges to determine if the strain-induced load requirement of $300 \mathrm{lbf}$ per wing at full scale could be met. The available parameters were discussed in section II-A and include changes to FLEXSEL cross

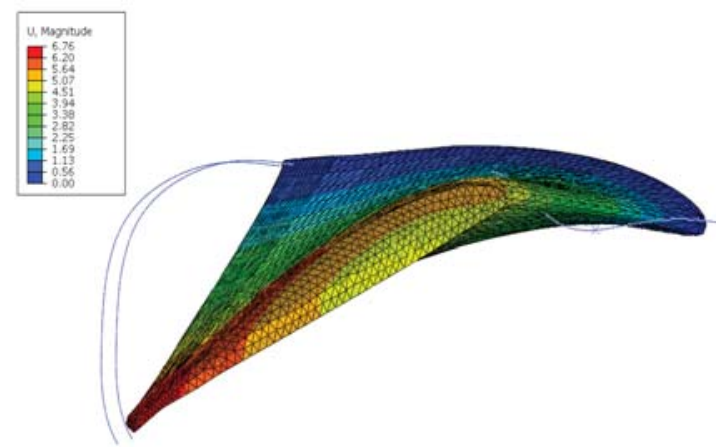

Figure 25. Displacement of solid-section FLEXSEL at full deployment.

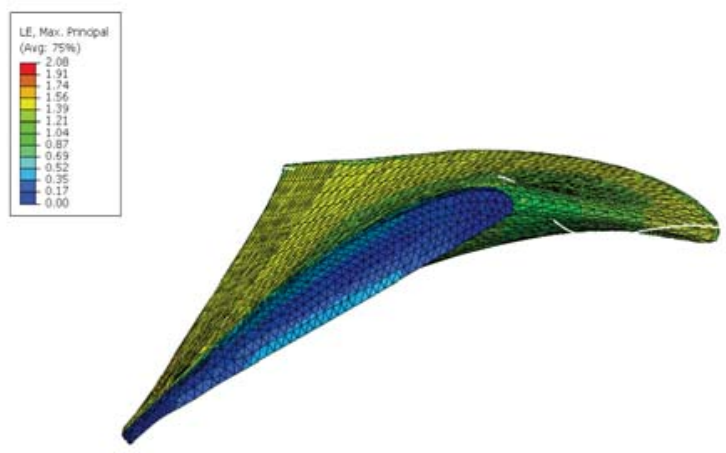

Figure 26. Logarithmic strain in solid-section FLEXSEL at full deployment.

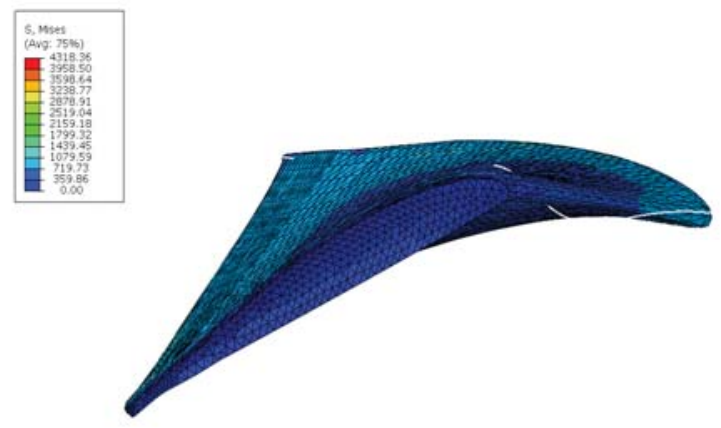

Figure 27. von Mises stress in solid-section FLEXSEL for EP1150 material at full deployment. section, span and reference, stress-free configuration. Results of that study are given in this section.

The detailed FE model described in the previous section was highly computationally intensive. Furthermore, it was found that simplifications to the interaction between the elastomer and the inserts were necessary to achieve simulation to full flap deployment. The solid-section FLEXSEL model was further simplified, as described in section III-E., which entailed elimination of the fitting and insert hardware from the model and imposition of the enforced flap motion directly on the elastomeric attachment. Although significant differences in the deformation field and resultant force were observed between the detailed and simplified models, the deformation response in the unsupported area was relatively consistent. The resultant force was similar but greater for the simplified model, which was expected due to reduced compliance at the boundary. Finally, trends observed in initial simulations with the simplified model were consistent with those from the detailed model. Thus, study of parameters to reduce the force required to deform the FLEXSEL was conducted with the simplified FE model.

\section{A. Solid Section}

A simplified FE model was developed for the baseline FLEXSEL concept having a solid section of the EP1150 material. The model consisted of 46,748 tetrahedral hybrid elements (C3D4H). Displacement, logarithmic strain and von Mises stress fields resulting from reconfiguration of the FLEXSEL structure from the retracted to deployed positions are shown in Figure 25 - Figure 27. It can be seen that particularly high logarithmic strains, on the order of 1.6 or equivalently a linear strain of $\sim 500 \%$, occur near the trailing edge of the structure. The free-body reaction force resulting from the stress distribution was $\sim 5,207$ lbf for the EP1150 material. Note that this is greater than the force evaluated from the detailed FE model, as described in section IV-E. Recall that the EP1150 constitutive response was considered to be stiff in the relevant range of materials. A material change from EP1150 to the vulcanized rubber in the model just described reduced the free-body reaction force to $\sim 1548 \mathrm{lbf}$, as shown in Figure 28. Although this is an enormous reduction, the force required to deform the solid section of vulcanized rubber remains over an order of magnitude too high. 


\section{B. Hollow Section}

Initially, a foam-core concept was considered a viable approach to soften the constitutive response of the material in the cross section while retaining structural form to help maintain an aerodynamic shape and resist wrinkling during deformation. However, as reported in the Material Considerations section (II-B.), known elastomeric foam materials were found to exhibit stiffer material response in tension than homogeneous elastomers of interest. Thus, known elastomeric foams are not useful for force reduction. A hollow FLEXSEL concept was considered as the limiting case. The hollow section configuration is shown in Figure 29, where it can be seen that the cross section remaining in the unsupported span has been drastically reduced, i.e., to a thin skin. The thickness of the elastomeric skin was $\sim 0.050$ inches, which was considered as minimum gauge because of fabrication and failure concerns. With a perimeter of approximately 10 inches $\times 2$, the resulting cross-sectional area was about $1 \mathrm{in}^{2}$. A FE model was developed for this structure and results from simulation of the flap movement are shown in Figure 30. Lateral and rear views are shown, along with a section view at approximately mid-span, of the deformed configuration. Some mild wrinkling of the elastomericskin surface can be seen, but it is probably acceptable from aerodynamic performance considerations. The free-body resultant force for this case with the EP1150 material was $\sim 500 \mathrm{lbf}$ (see Figure 31). Additional reduction in the force required for deformation can be achieved with a material change, as in the solid-section case. However, the force would still be relatively high at $\sim 240 \mathrm{lbf}$ for this model scale.

\section{Extended Span, Hollow Section}

Computational aeroacoustics results suggested that an increase in span of the FLEXSEL concept would still maintain the projected aeroacoustic and aerodynamic benefit. However, the allowable span increase was a modest $46 \%$, increasing from 1.5 inches to 2.2 inches in unsupported span at model scale. Other than the increased span, the FE model for this concept (Figure 32) was equivalent to that for the hollow case at the baseline span length in the previous section. Analysis of this case to full flap deployment resulted in a free-body reaction force of $\sim 320 \mathrm{lbf}$ for the EP1150 material and $\sim 117 \mathrm{lbf}$ for the vulcanized rubber material (Figure 33). This force for rubber was considered to be within a factor of 2 of the desired result at model scale. It is also noted that the wrinkles exhibited in the deformed configuration were slightly less prominent in this case relative to the baseline-span case.

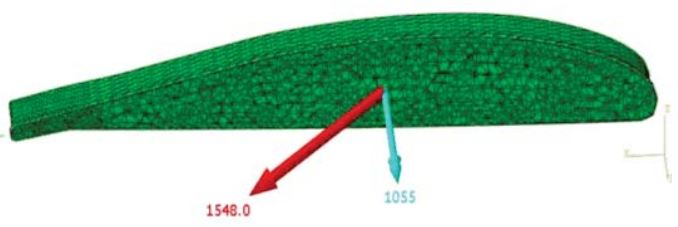

Figure 28. Free-body resultant force for solid-section FLEXSEL with rubber material at full deployment.

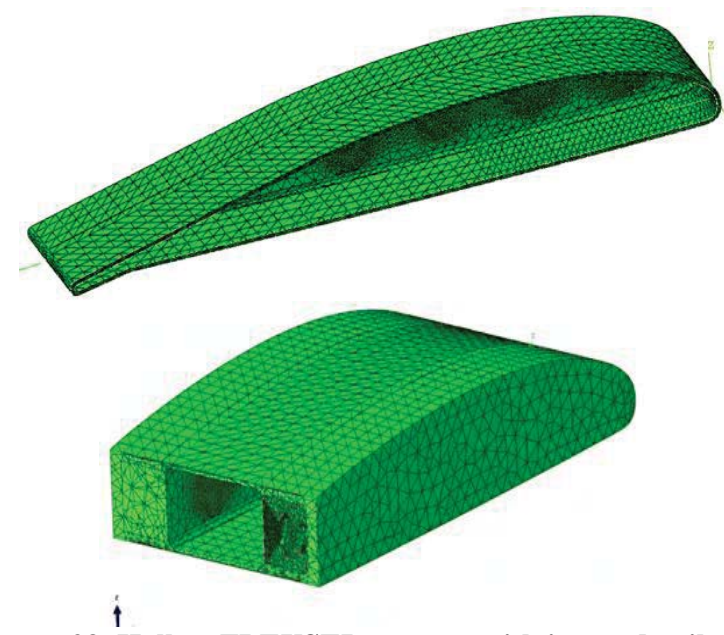

Figure 29. Hollow FLEXSEL concept with insert details

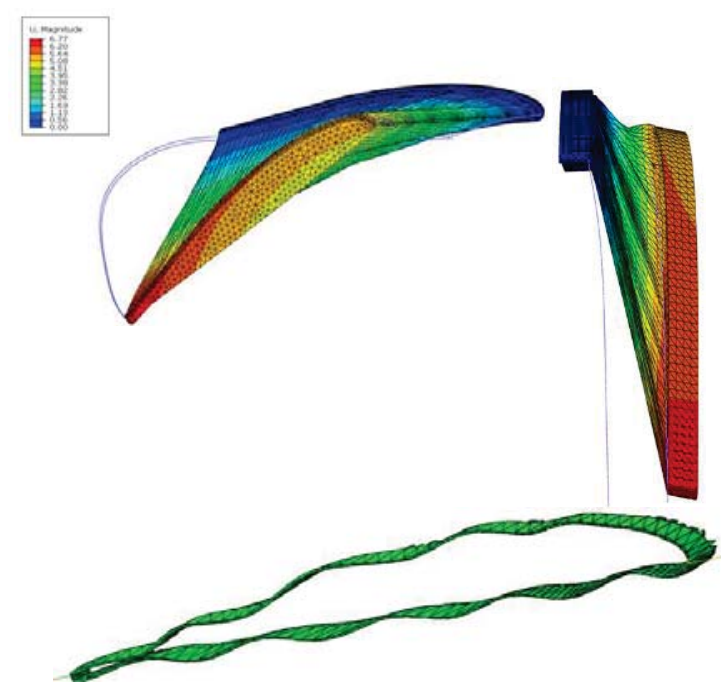

Figure 30. Deformed shape of the hollow FLEXSEL concept at full deployment. 


\section{FLEXSEL Deployment from a Partial-Flap Setting}

Additional reduction in the deformation-induced load of the FLEXSEL structure was required for implementation feasibility. Thus, deployment from a stress-free shape at an intermediate flap position was considered to reduce the amount of deformation required to achieve the fullydeployed flap position.

A contrived, intermediate, stress-free shape was developed as follows. Deployment of the flap with the extended-span, hollow FLEXSEL concept was simulated from the retracted position (i.e., flap 0) to a deformed configuration roughly halfway through the flap movement (Figure 34a). The stress and strain fields in the FLEXSEL structure were then reset to zero (Figure 34b). Note that some wrinkling was present in the new reference, stress-free configuration at approximately half-flap. A new analysis was conducted to predict the deformation behavior and force developed in the structure during deployment to the full-flap (flap 39) configuration (Figure 34c). The fullydeployed structure was returned to the intermediate, stress-free configuration and zero stress and strain fields were confirmed. Finally, flap movement to the fully-retracted configuration was simulated in order to study the deformation and force developed between flap 20 and flap 0.

The free-body resultant force, moment and approximate flap angles during the course of these simulations are shown in Figure 35 and Figure 36. It can be seen that the force required to deform the FLEXSEL structure from approximately half-flap to fullflap was $\sim 35 \mathrm{lbf}$ (Figure 36). Further reduction in the force was achieved with a switch to the vulcanized rubber material, which resulted in a force of $\sim 23 \mathrm{lbf}$. Although this force to reach full deployment is well within the acceptable limit at model scale, the force required to return from half-flap (stress free) to the fully-retracted position was $\sim 60$ lbf (Figure 36), just outside of the acceptable range. However, retraction also produced deformation due to buckling of the elastomeric skin that would result in unacceptable aerodynamic performance.

Further analysis was performed by changing the intermediate, stress-free flap angle. Two other intermediate flap settings considered were approximately $25 \%$ and $75 \%$ positions in the flap movement. It was apparent that the amount of force required for deployment and retraction can be adjusted

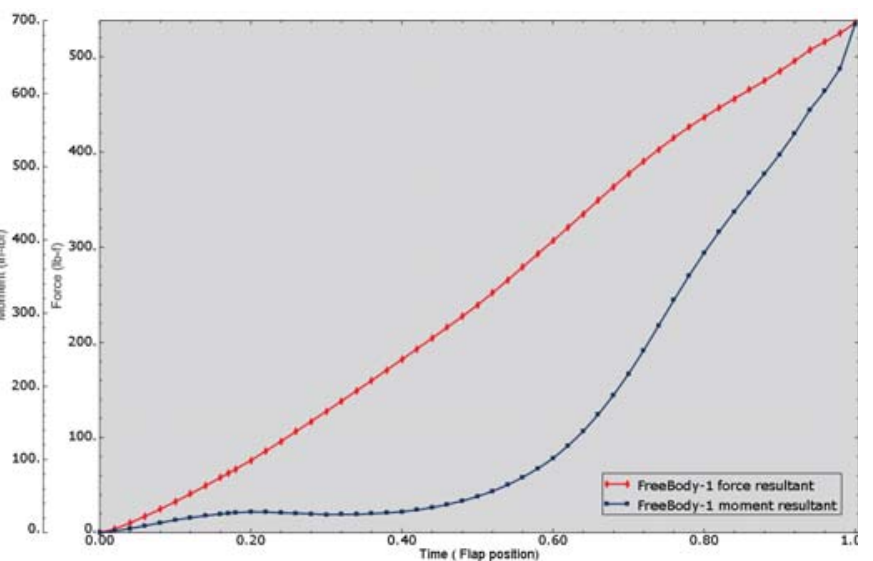

Figure 31. Free-body force/moment response of the hollow FLEXSEL concept to full deployment.

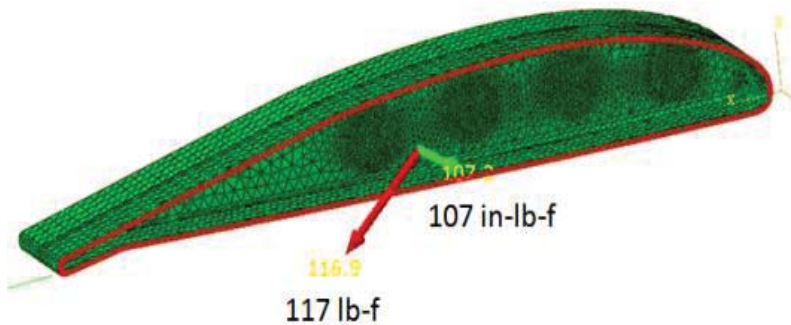

Figure 32. Free-body resultant force for extended-span, hollow FLEXSEL with rubber at full deployment.

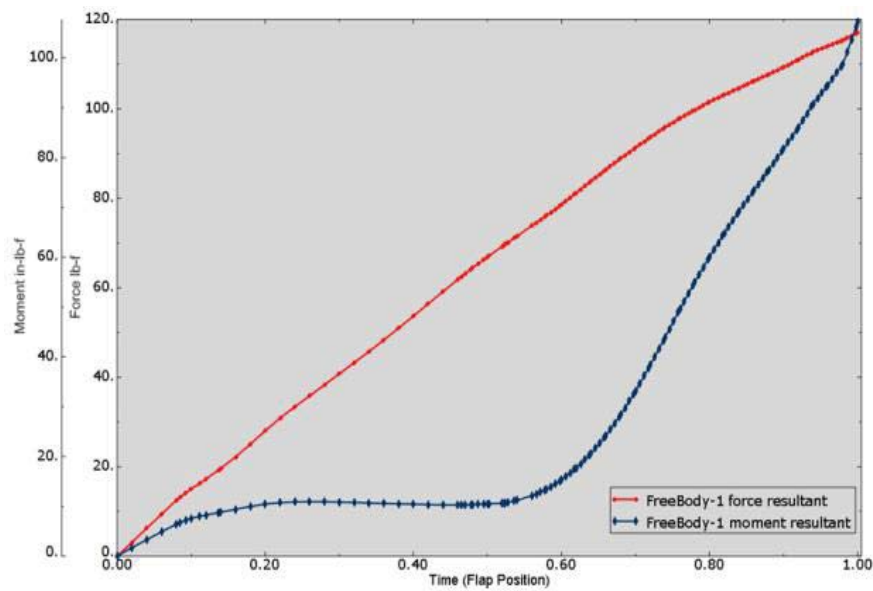

Figure 33. Free-body force/moment response of the extended-span, hollow FLEXSEL to full deployment. and balanced, since these forces work in opposition depending on whether strain energy is stored or released. 
Buckling of the elastomeric skin occurred in all cases when returning to the fully-retracted configuration. Further analysis and design studies are required to eliminate the post-buckling deflections. Another concept under consideration involves determining a compressed (stressed) configuration for the retracted position that does not buckle the structure and that can be stress-relieved during deformation to the intermediate, stress-free configuration. The deformation-induced-force results from various FLEXSEL concepts analyzed are summarized in Table 1.

\section{Conclusion}

Airframe noise is recognized as an environmental problem of increasing importance because of tightening noise regulations and progress in engine noise reduction. Flap side edge noise is a prominent component of airframe noise. A promising treatment for flap side edge noise is a structural connection or link between the main wing and flap side edges that delays and weakens the vortex system responsible for flap noise. A gradually-faired, structural link with significant span-wise dimension was previously proposed and is intuitively appealing, but requires satisfaction of extremely disparate requirements of sustaining high aerodynamic loads while remaining highly deformable. The approach taken here was minimization of the span-wise dimension for such a structural link, thereby minimizing the aerodynamic load on the link at the expense of requiring increased deformation from the imposed flap motion. The resulting concept was termed the FLEXible Side Edge Link (FLEXSEL) concept, which is highly deformable and enabled by hyperelastic materials, atypical structural configurations and novel structural integration approaches.

The design for a static (non-deforming), wind-tunnel model, representing the baseline FLEXSEL concept, was previously developed and included a configuration consisting of an aerodynamically-shaped, elastomeric link of solid cross section and detailed airframe integration features. Initial hand calculations indicated that the baseline FLEXSEL concept required the excessive force to deform the link through the flap motion. The objective of this work was to perform a computational study over known parameters to render the deformation-induced force acceptable and satisfy other requirements necessary for practical implementation of the FLEXSEL concept. Computational studies were performed on deformable configurations using selected elastomer materials with known constitutive properties. These studies required complex finite element analysis of evolving FLEXSEL concept variants. Analyses of these concepts were very challenging because of material and geometric nonlinearities, very large deformations, material and deformation discontinuities, atypical material responses and intricate geometric details. A building block approach for simulation of the detailed elastomeric assembly was pursued performing simulation with simplified elastomer components, and simulations with isolated attachement fitting and inserts where necessary to demonstrate responses of the detailed assembly.

Variants of the baseline FLEXSEL configuration were aimed at reducing the force required to deform the structure and included 1) reducing the material and structural stiffness and 2) reducing the deformation by increasing the span of the structure and devising a stress-free shape at an intermediate flap position. An elastomeric-foam-core configuration was considered and trends suggested that known elastomeric-foam materials would not provide the intended stiffness reduction and shape retention properties because of their stiff constitutive response in tension, compared to homogeneous elastomers, and excessively soft constitutive response in compression. A hollow-core concept was evaluated as an immediate alternative to the foam-core configuration, so as to get a lower-bound estimate of deformation-induced force. The hollow core provided a significant reduction in stiffness and deformation-induced force required for operation of the FLEXSEL structure, but with some wrinkling due to asymmetrical geometry and loading were seen in the thin elastomeric skin. Further reduction in the deformationinduced force was achieved with a configuration having an increased span. Stress-free configurations at intermediate flap positions were studied as alternatives to the baseline, stress-free-retracted configuration. The results showed that additional reductions in the deformation-induced force can be achieved, but there is a tradeoff with undesirable buckling deformation seen during retraction from the intermediate-stress-free position. Although the deformationinduced force requirement was met by a combination of the hollow core, extended span and intermediate stress-free shape variants of the FLEXSEL design, other challenges remained including some buckling deformation during retraction that could be undesirable for aerodynamic performance. Also, trends from the computational analysis results suggest that further improvement can be achieved. In this regard, the use of formal mathematical optimization techniques with toplogical variables holds a promising path forward to establish design of a practical FLEXSEL structure to meet all requirements. The design optimization and experimental verification of the optimized design is left as future work. 

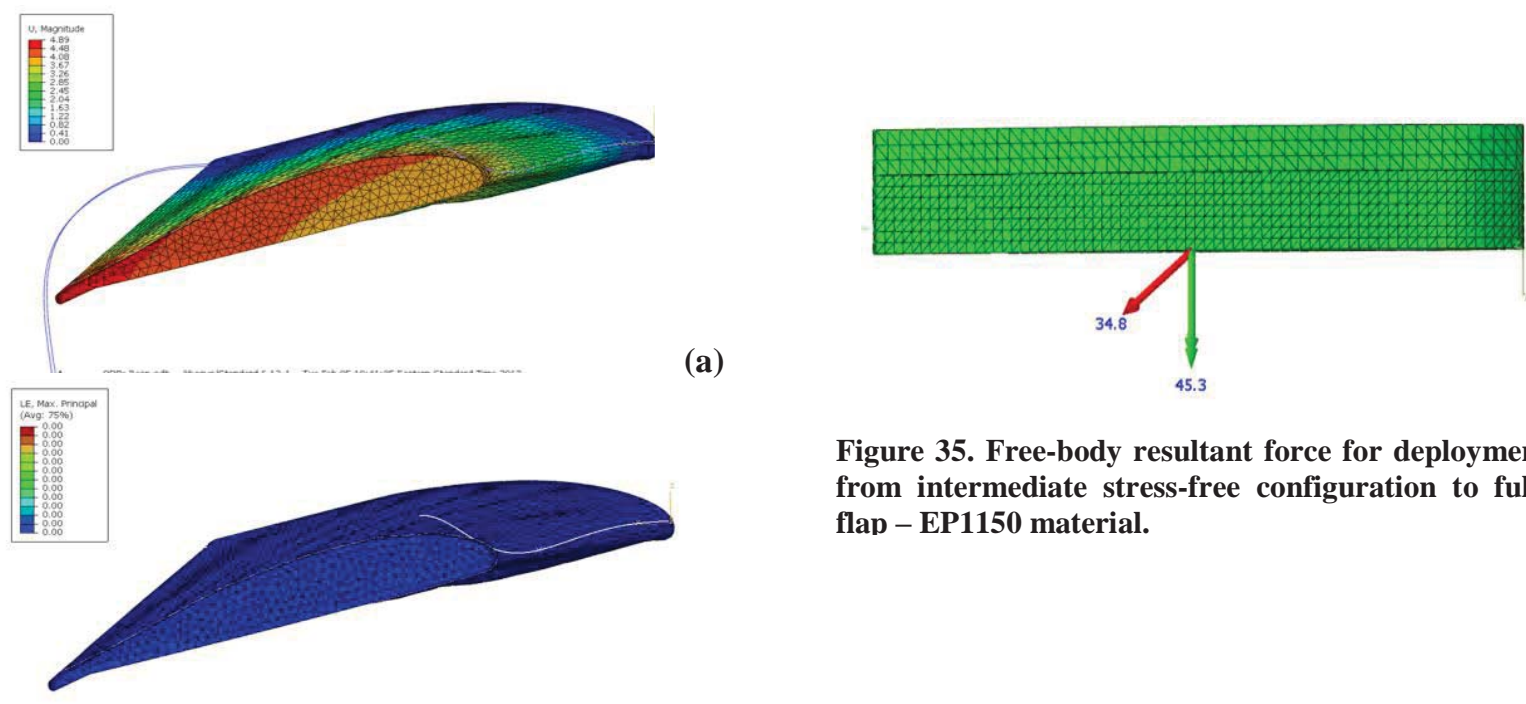

Figure 35. Free-body resultant force for deployment from intermediate stress-free configuration to fullflap - EP1150 material.

(b)

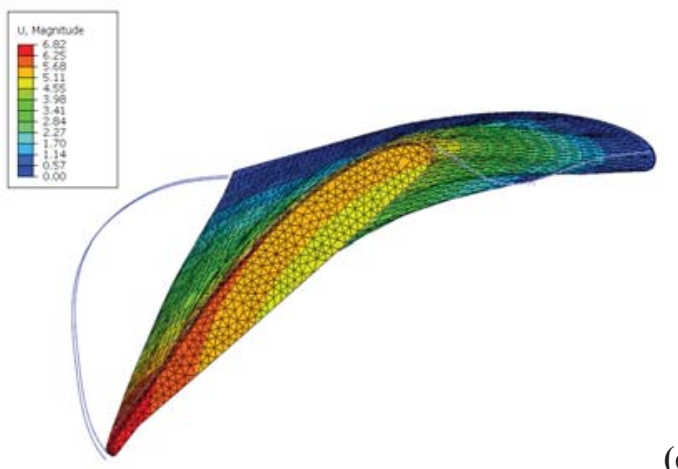

(c)

Figure 34. FLEXSEL deployment from intermediate stress-free sequence (a) deformation at half-flap position, (b) reset of stress and strain fields at half-flap to establish new reference condition, and (c) deployment to full-flap position.

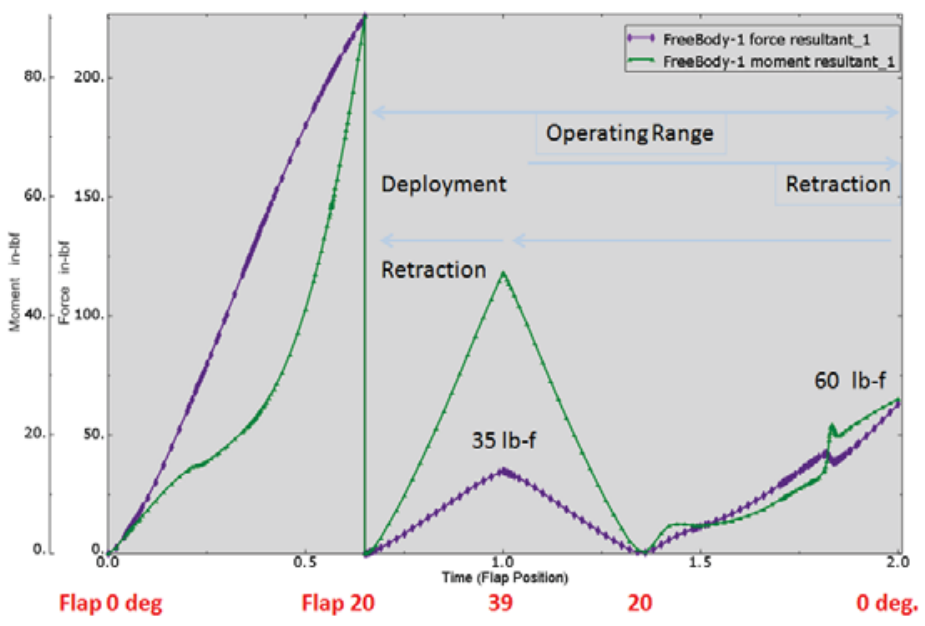

Figure 36. Force and moment resultants corresponding to contrived, intermediate stress-free case. 
Table 1 Deformation-induced force predicted for various FLEXSEL concepts.

\begin{tabular}{|c|l|c|}
\hline $\begin{array}{c}\text { Concept } \\
\text { ID }\end{array}$ & Description of the Elastomeric Concept & $\begin{array}{c}\text { Max. Stretch Force } \\
\text { at 39 deg Flap, } \\
\text { lb-f. }\end{array}$ \\
\hline 1 & Solid Aerofoil Block - EP1150 Material & High \\
\hline 2 & Hollow Core - EP1150, Flap 0 to 39 deg. & 535 \\
\hline 3 & Hollow Core - Rubber, Flap 0 to 39 deg. & 241 \\
\hline 4 & Hollow Core, Ext. Span - EP 1150, Flap 0 to 39 deg. & 320 \\
\hline 5 & Hollow Core, Ext. Span - Rubber, Flap 0 to 39 deg. & 117 \\
\hline 6 & Hollow Core, Ext. Span - EP 1150 Flap 20 to 39 deg. & 35 \\
\hline 7 & Hollow Core, Ext. Span - EP 1150 Flap 13 to 39 deg. & 102 \\
\hline 8 & Hollow Core, Ext. Span - EP 1150 Flap 28 to 39 deg. & 21 \\
\hline
\end{tabular}

\section{Acknowledgments}

The guidance provided by Mehdi Khorrami, David Lockard, and John Lin (NASA LaRC) on requirements of the application and on aerodynamics/aeroacoustics in general is greatly appreciated. The assistance of Mark Griffith, Robert Andrews and Gary Wainwright (NASA LARC) in specimen fabrication, C. Edward Townsley (NASA LaRC) and Stewart Walker (AMA Inc.) in specimen testing, and Kris Wise (NASA LaRC) and Marisabel LebronColon (NASA GRC) in material surveys is also gratefully acknowledged. Support from the NASA's Fixed Wing and Environmentally Responsible Aviation Projects is also gratefully acknowledged.

\section{References}

${ }^{1}$ Lockard, D. P., “Airframe Noise Research Overview” Acoustics Technical Working Group Meeting, NASA Glenn Research Center, April 23-24, 2013

${ }^{2}$ Washburn, A., “An Overview of NASA's Environmentally Responsible Aviation Project Plan: 2013-2015,” 51 ${ }^{\text {st }}$ AIAA Aerospace Sciences Meeting, Grapevine, TX, Jan. 07, 2013.

${ }^{3}$ Streett, C. L., Casper, J. H., Lockard, D. P., Khorrami, M. R., Stoker, R.W., Elkoby, R., Wenneman W. F., Underbrink, J. R., "Aerodynamic Noise Reduction for High-Lift Devices on a Swept Wing Model," 44 ${ }^{\text {th }}$ AIAA Aerospace Science Meeting and Exhibit, Reno Nevada, AIAA 2006-212, 2006.

${ }^{4}$ Khorrami, M. R., Lockard, D. P., Humphreys, Jr., W. M., Choudhari, M. M., Van de Ven, T., "Preliminary Analysis of Acoustic Measurements from the NASA-Gulfstream Airframe Noise Flight Test," $14^{\text {th }}$ AIAA/CEAS Aeroacoustics Conference (29th AIAA Aeroacoustics Conference), Vancouver, British Columbia Canada, Paper No. AIAA 2008-2814, 5 - 7 May 2008.

${ }^{5}$ Khorrami, M. R., and Neuhart, D. H, "Aeroacoustic Study of a High-Fidelity Aircraft Model: Part 2-Unsteady Surface Pressures," 18th AIAA/CEAS Aeroacoustics Conference (33rd AIAA Aeroacoustics Conference), Colorado Springs, CO, AIAA 2012-2234, 04 - 06 June 2012.

${ }^{6}$ Davidson, M. E., “Continuous Moldline Link (CML),” NASA Contractor Informal report, Contract NAS1-00086, Task NNL04AB77T, Boeing IDS- Advanced Structures, March 27, 2006.

7“Flexible Friend - Shape Changing Wing Promises Cleaner and Quieter Flights," Aviation Week and Space Technology, January 28, 2013, Page 13.

8“Adaptive Compliant Trailing Edge Project,” This Week at NASA, April 26,2013,

http://www.nasa.gov/multimedia/videogallery/index.html?media_id=162385381

${ }^{9} \mathrm{Su}$, J., "Mechanical (tension) Test of Elastomeric Materials for Fenced Flap Concept," NASA Langley, Hampton, VA, September 30, 2009 (unpublished).

${ }^{10}$ Treloar, L. R. G., "Stress-Strain Data for Vulcanised Rubber Under Various Types of Deformation," Transactions of the Faraday Society, vol. 40, pp. 59-70, 1940.

${ }^{11}$ ABAQUS User's Manual, Version 6.12-1, Dassault Systemes SIMULIA Corp, Providence, RI, USA, 2012

${ }^{12}$ Dai, Xi, Sabuwala, T., Gioia. G., "Experiments on Elastic Polyether Polyurethane Foams Under Multiaxial Loading: Mechanical Response and Strain Fields,” Journal of Applied Mechanics, Vol. 78 / 031018-1 to 11, May 2011.

13،Flexible Polyurethane Foam, EF 4005," Technical Data Sheet Downloads, 10/17/2012. General Plastics Manufacturing Co., URL: http://www.generalplastics.com/downloads/tds/ef-4005-tds.pdf [cited May 6, 2013]. 